\title{
THE EARLIEST ATMOSPHERE OF THE EARTH
}

\author{
JAMES C.G. WALKER \\ Space Physics Research Laboratory, University of Michigan, Ann Arbor, MI 48109 \\ (U.S.A.)
}

(Received July 27, 1981; revision accepted October 19, 1981)

\section{ABSTRACT}

Walker, J.C.G., 1982. The earliest atmosphere of the Earth. Precambrian Res., 17: $147-171$.

This paper examines, from the point of view of atmospheric physics, the possibility that the initial complement of volatiles in the material that formed the Earth was very much larger than that remaining. The escape of large amounts of hydrogen into an interplanetary vacuum is possible only if the heat source in the upper levels of the atmosphere, provided by solar extreme ultraviolet and particle emissions, was much larger than it is today. Rapid escape of hydrogen would have swept heavy minor gases along with it. If the rate of escape declined due to a decreasing upper atmospheric energy source, minor gases would have been selectively retained by the Earth, beginning with the heaviest. Rotation would not have facilitated escape significantly because a rapidlyescaping atmosphere would have tended to conserve angular momentum.

If Earth accreted in the presence of a gaseous nebula it would have acquired a dense, hot atmosphere of nebular composition. This primary atmosphere would probably have been convective and adiabatic at lower levels, with the upper atmosphere approximately isothermal and devoid of turbulence. Planetary rotation would have imposed a global circulation on the upper atmosphere and overlying nebula with downwelling over the poles and upwelling over the equator. This circulation would have carried away into the nebula any gases released from the solid phase during the course of accretion. The primary atmosphere may not have dissipated completely after the disappearance of the nebula. Heavy gases particularly may have been retained by the Earth to contribute to the present volatile inventory.

\section{INTRODUCTION}

The bulk composition of the Earth is depleted, relative to the solar system, in elements that form volatile chemical compounds. In the case of the inert gases that depletion has been known for a long time (Moulton, 1905; Aston, 1924; Russell and Menzel, 1933; Suess, 1949; Brown, 1952; Sagan, 1967; Meadows, 1973; Anders and Owen, 1977). More recent interpretation of geochemical and geophysical results has established that the phenomenon extends also to rock-forming elements (see Ringwood, 1979) and is completely regular, in the sense that deficiencies in terrestrial abundances correlate with the volatilities of the chemical compounds that can be 
expected to have formed in a gas of solar system composition (the solar nebula).

The interpretation of these chemical data is clear. The Earth formed predominantly from solid constituents of the solar nebula, incorporating little or no gaseous material.

This fundamental concept can be elaborated in a number of ways. One possibility is that the nebula in the vicinity of the Earth was initially heated by compression to temperatures near $2000^{\circ} \mathrm{C}$, high enough to vaporize all constituents. As the nebula cooled, solids condensed in a progression of increasing volatility (Grossman, 1972; Grossman and Larimer, 1974). After a period of time, this process was halted by decreasing pressure of the nebular gas (the dispersal of the nebula). The Earth was formed by the accretion of solid planetesimals that had condensed at different temperatures in the cooling solar nebula. This model can yield an initial Earth that is either homogeneous or radially-zoned (heterogeneous) in chemical composition, depending on the relative time scales of cooling and accretion. If accretion is relatively rapid, the innermost regions of the Earth will incorporate refractory material only, and successively less-refractory material will be progressively added to the growing Earth as it condenses from the cooling solar nebula (Turekian and Clark, 1969, 1975; Clark et al., 1972).

Alternatively, if accretion is slow relative to cooling, the Earth will form with a composition initially independent of radius out of a mixture of highand low-temperature condensates.

Another point of view puts more emphasis on spatial rather than temporal gradients in temperature. A model developed by Lewis (1972a,b; $1974 a, b)$, for example, attributes differences in the bulk properties of the inner planets and in the satellites of the outer planets to a decrease of temperature in the nebula with increasing distance from the Sun (or the primary in the case of satellite systems). In the simplest version of this theory, the planets formed from solid material that was in thermodynamic equilibrium with nebular gas at the temperature appropriate to the planet's distance from the Sun. Thus, more distant planets and especially their satellites incorporated more volatile material. Gravitational perturbations of planetesimal trajectories could cause some spread in the equilibration temperatures of the material incorporated by a given planet.

The temperature gradient hypothesis has been further developed by Ringwood (1979), who attaches importance to a decrease in temperature with distance normal to the ecliptic plane. Ringwood suggests that a planet can accrete a mixture of refractory material formed under high-temperature conditions close to the ecliptic plane and volatile-rich low-temperature condensates formed simultaneously at some distance from the ecliptic plane. The time for solid planetesimals to settle, under the action of gravity, through the nebular gas to the ecliptic plane, where they can be incorporated into a growing planet, is very short (Goldreich and Ward, 1973; Hills, 1973; Weidenschilling, 1974, 1976). 
There exist convincing geochemical arguments for the formation of the Earth from a mixture of high- and low-temperature condensates (see Ringwood, 1979), and such a mixture is consistent with all of the condensation hypotheses described. As far as the subsequent history of the Earth and the atmosphere is concerned, it probably matters little whether planetesimals of different volatilities condensed in different parts of the nebula at the same time or in the same part at different times. However, Ringwood (1979) has argued that our present understanding of the internal constitution and structure of the Earth is not consistent with its accretion in a form initially zoned with respect to the volatility of its constituents, i.e. refractories in the center and volatile-rich material near the surface. For the time being, at least, the heterogeneous accretion hypothesis is in eclipse.

An early theory, advanced by Urey (1952) and others, was that the process of accretion of planetesimals into planets was relatively slow and occurred, at least in the inner solar system, after the disappearance of the gaseous nebula (see Jastrow and Cameron, 1963). The rate of release of gravitational energy during the course of accretion was supposed to have been sufficiently slow to permit an initially cold Earth to form, devoid of atmosphere, and without large-scale heterogeneities of composition (MacDonald, 1959). Radioactive heating of the interior was supposed to have raised internal temperatures (and lowered viscosity) over a period of time to the point where differentiation on the basis of density became possible. The release of gravitational energy associated with internal differentiation (formation of the core) raised much of the Earth to high temperatures and presumably caused the release of the more volatile constituents to the atmosphere (degassing). The distinguishing features of this model, which at one time attracted a lot of attention (see Brancazio and Cameron, 1964) are the late occurrences, well after accretion and nebular dissipation, of core formation and degassing. The atmosphere and ocean, according to this point of view, are entirely secondary, having been released by tectonic and differentiation processes from an originally cold and airless planet.

A minor modification of this model would allow accretion to occur in the presence of a gaseous nebula. The planet would therefore initially be surrounded by a gravitationally-concentrated atmosphere of solar system composition (a primary atmosphere). Earth's present-day inventory of atmophile (atmosphere-forming) constituents is so different in composition from that of the solar system that very little of this primary atmosphere can have survived (see Walker, 1977; Pollack and Yung, 1980). It is presumed to have escaped to space, largely or completely, before degassing of the secondary atmosphere began.

If it is now assumed that Earth's original complement of volatiles has been little modified by subsequent loss to space it is possible to infer the bulk composition of the mixture of materials that accreted to form the Earth. This assumption has been made implicitly or explicitly in nearly all 
studies of the origin of the atmosphere. Recent examples of the approach are by Turekian and Clark (1975), Anders and Owen (1977), Walker (1977) and Pollack and Black (1979). The procedure, as far as atmophile elements is concerned, is to conduct a careful survey of the Earth's present inventory, including estimates of the volatile abundances in the crust and upper mantle, correct for the loss of hydrogen and helium to space and for the radiogenic production of helium and argon, and compare the result with the composition of one or more classes of meteorite.

The purpose of this paper is to explore the possibility that this assumption is incorrect. Is it possible that the Earth's endowment of volatiles was originally much larger than it is today, and that most of this initial endowment has been lost to space? The question has been raised most forcibly by Ringwood (1979), who argues on geochemical grounds that a significant fraction $(10-15 \%)$ of the material that accreted to form the Earth was lowtemperature condensate, rich in volatiles. Ringwood's arguments and evidence are sophisticated and diverse, but the line that possibly bears most strongly on the volatile inventory is as follows. The degree of oxidation of iron in the mantle (but see Arculus and Delano, 1980) and the possible incorporation of oxygen in solution in the core implies a large source of oxygen, most of which must have been accreted in the form of water of hydration in material resembling $\mathrm{C} 1$ carbonaceous chondrites. According to Ringwood's model, the water reacted with iron at high temperatures during the course of accretion to yield hydrogen and iron oxide. The hydrogen subsequently escaped to space, carrying other volatile elements with it.

The initial inventory of atmophile elements implied by this model is compared with Earth's current endowment in Table I. The data are from Turekian and Clark (1975) adjusted to Ringwood's (1979) proposal that 15\% of the mass accreted by the Earth was low-temperature condensate containing $20 \%$ water. These data suggest that the Earth has lost nearly all its hydrogen, carbon and nitrogen and a substantially smaller fraction of its

\section{TABLE I}

Volatile inventories on the Earth (g atom)

\begin{tabular}{llll}
\hline & $\begin{array}{l}\text { Accreted } \\
\text { (a) }\end{array}$ & $\begin{array}{l}\text { Preserved } \\
\text { (b) }\end{array}$ & $\begin{array}{l}\text { Preservation factor } \\
\text { (b/a) }\end{array}$ \\
\hline $\mathrm{H}$ & $2 \times 10^{25}$ & $3.6 \times 10^{23}$ & 0.018 \\
$\mathrm{C}$ & $2.5 \times 10^{24}$ & $2.3 \times 10^{22}$ & 0.009 \\
$\mathrm{~N}$ & $2 \times 10^{23}$ & $1.0 \times 10^{21}$ & 0.005 \\
$\mathrm{Ne}$ & $9 \times 10^{15}$ & $3.3 \times 10^{15}$ & 0.4 \\
${ }^{36} \mathrm{Ar}$ & $3 \times 10^{16}$ & $5.7 \times 10^{15}$ & 0.2 \\
$\mathrm{Kr}$ & $4 \times 10^{14}$ & $2.1 \times 10^{14}$ & 0.5 \\
$\mathrm{Xe}$ & $3 \times 10^{14}$ & $1.7 \times 10^{14}$ & 0.6
\end{tabular}

Data from Turekian and Clark (1975) and Ringwood (1979). 
inert gases. Whether the apparently enhanced retention of inert gases is to be taken seriously is not clear. Ringwood devotes relatively little attention to atmophile elements and it is possible that I have interpreted his model too literally with respect to the inert gases. There is little room for misinterpretation with respect to carbon and hydrogen, however. If Ringwood's model is correct, the Earth has lost an enormous amount of these elements during the course of, or subsequent to, its accretion. Under what circumstances could this loss have occurred?

The possibility exists that the present atmosphere and oceans are a small remnant of an originally much more massive atmosphere. Is it also possible that the atmosphere is not secondary, as has been supposed, but a primary atmosphere profoundly modified by degassing and escape of much of its mass to space? This possibility arises from new ideas concerning the relative timing of accretion, degassing and dispersal of the gaseous nebula.

The old idea of an initially cold Earth has fallen from favor (Hanks and Anderson, 1969; Fanale, 1971). Many studies adopting a variety of different points of view have concluded that the time scale for accretion was short and that accretional heating was sufficient to produce an initially hot Earth of low enough viscosity to permit solid-state convection in the interior. The early thermal history of the Earth has been examined by Stevenson (1982) in a very illuminating paper. He concludes that formation of the core occurred during the course of accretion and that most of the outgassing and differentiation of the mantle occurred as a transient response to accretional heating and within no more than a few hundreds of millions of years of the time of accretion. These views are entirely in accord with the requirements of Ringwood's model.

What, then, are the possible scenarios?

(1) The Earth accreted after dispersal of the gaseous nebula. It therefore never had a primary atmosphere. The present atmosphere is entirely secondary, although it may be a small remnant of an originally massive secondary atmosphere. This scenario may contradict the presumed short time scale for accretion.

(2) The Earth accreted in the presence of nebular gases, which were concentrated by gravity in the vicinity of the Earth to provide a primary atmosphere. To this primary atmosphere were added gases released from the solid phase during the course of accretion and shortly thereafter. Most of this mixed primary and secondary atmosphere was lost at the time of dispersal of the gaseous nebula or soon after, and the present atmosphere is a remnant, possibly augmented by subsequent degassing.

(3) This scenario is a limiting case of (2) above. All of the mixed primary and secondary atmosphere was lost, and the present atmosphere is entirely a product of subsequent degassing. 
I am not able to offer a self-consistent and plausible model that would choose between these scenarios on the basis of data. Instead, I propose to explore some of the implications of one or another scenario in the expectation that basic atmospheric physics can impose some constraint on speculation in this field.

\section{ESCAPE: HYDRODYNAMIC VERSUS KINETIC FORMULATIONS}

A gravitationally-bound atmosphere at finite temperature must lose mass into the surrounding vacuum of space (see Walker, 1977), although the rate of this loss can be negligibly small if the average kinetic energy of the gas molecules is much less than their gravitational potential energy. Two theoret. ical approaches have been developed for describing the escape of atmospheric gases. One, based on the kinetic theory of gases, yields results frequently referred to as 'Jeans escape' (Hunten and Donahue, 1976). The other, hydrodynamic approach, yields results sometimes described as 'blow-off' (Hunten, 1973). Jeans escape and blow-off do not refer to different physical processes. They refer to the results of different theories that approximately describe the same process. The hydrodynamic theory is approximate, in that it neglects departures from continuum behavior of the gas at high altitudes, where the mean free path between molecular collisions becomes very large. The kinetic theory neglects distortions of the velocity distribution of the molecules caused by mass motion at the level of transition from continuum to free molecular flow (Chamberlain, 1963, 1969; Hays and Liu, 1965; Chamberlain and Campbell, 1967; Chamberlain and Smith, 1971; Brinkmann, 1970, 1971).

Which approximate theory provides a more accurate description of the escape process depends on conditions. If the escape rate is large, the hydrodynamic theory is more nearly correct. Jeans escape provides a better description of small escape rates. The distinction between small and large escape rates can be made on the basis of either of two equivalent criteria.

Atmospheric density, under the action of gravity, decreases more or less exponentially with altitude (see Goody and Walker, 1972). The mean free path between collisions therefore increases. At high altitudes, the mean free path becomes equal to and then exceeds the characteristic distance for significant density change (called the 'scale height', this characteristic distance is the negative reciprocal of the natural logarithm of the density gradient). The level of this transition is called the exobase. It marks a transition from continuum dynamics to free molecular flow (see Walker, 1977).

As the density of an escaping gas decreases with altitude its flow velocity must increase in order to maintain a constant flux. At high altitudes the flow velocity becomes equal to and then exceeds the speed of sound. The level of transition from subsonic to supersonic flow may be called the 'sonic level'. If the sonic level lies above the exobase, escape is best described by the kinetic theory of gases. Conversely, the hydrodynamic 
theory is most appropriate when the sonic level is below the exobase. So, the choice of escape theory can be made either by evaluating the outflow velocity at the exobase and comparing it with the speed of sound or by evaluating the density at the sonic level and comparing it with the density at the exobase.

The application of these criteria can be illustrated by considering the escape of the very large amounts of primordial hydrogen implied by Ringwood's theory of Earth origin. According to Table I, some $10^{25} \mathrm{~g}$ atom of hydrogen are to be lost. The time scale is uncertain, but it is almost certainly less than $10^{9} \mathrm{y}$, the length of time preceding the beginning of the sedimentary rock record. I shall assume a time scale of $10^{8} \mathrm{y}$ by way of illustration. The required escape flux is therefore $4 \times 10^{14} \mathrm{H}_{\text {atoms }} \mathrm{cm}^{-2} \mathrm{~s}^{-1}$ The density at the exobase is given by

$N_{\mathrm{e}}=1 / \sigma H \mathrm{~cm}^{-3}$

where $\sigma \mathrm{cm}^{2}$ is the cross-section for collisions and $H=\mathrm{kT} / \mathrm{mg} \mathrm{cm}$ is the ambient atmospheric scale height, where $\mathrm{k}$ is Boltzmann's constant, $T$ is absolute temperature, $m$ is molecular weight, and $g$ is gravitational acceleration. At a temperature of $500 \mathrm{~K}$ the scale height of atomic hydrogen is $424 \mathrm{~km}$. A typical value of $\sigma$ is $10^{-15} \mathrm{~cm}^{2}$, so an approximate value of $N_{\mathrm{e}}$ is $2.4 \times 10^{7} \mathrm{~cm}^{-3}$. A higher temperature would proportionately reduce $N_{\mathrm{e}}$, while the use of $\mathrm{H}_{2}$ rather than $\mathrm{H}$ would double it, neglecting change in the collision cross section.

The outflow velocity at the exobase is calculated by dividing the flux by the density. It is $1.7 \times 10^{7} \mathrm{~cm} \mathrm{~s}^{-1}$. The sound speed is given by $(\gamma \mathrm{kT} / \mathrm{m})^{1 / 2}$ where $\gamma=5 / 3$ for an atomic gas. At $500 \mathrm{~K}$ for atomic hydrogen it is about $2.6 \times 10^{5} \mathrm{~cm} \mathrm{~s}^{-1}$. The flow is evidently supersonic at the exobase, a conclusion that would not be altered by a different choice of temperature, by consideration of $\mathrm{H}_{2}$ rather than $\mathrm{H}$, or by a different time scale less than $10^{9} \mathrm{y}$.

An estimate of the density at the sonic level is obtained by dividing the escape flux by the sound speed. It is $1.5 \times 10^{9} \mathrm{~cm}^{-3}$, much greater than the density at the exobase. So the sonic level lies well within the region of continuum flow and the escape problem is most appropriately treated in the hydrodynamic approximation. My cavalier disregard of the finer points of the calculation (e.g. both flux and gravitational acceleration vary inversely with the square of geocentric distance) is justified by the extreme uncertainty concerning conditions at the time and by the very wide margin in favor of hydrodynamic rather than Jeans escape.

\section{HYDRODYNAMIC ESCAPE: MASS FRACTIONATION VERSUS BLOW-OFF}

The kinetic theory formulation of escape involves the fraction of molecules in a Maxwellian velocity distribution with velocities in excess of the 
escape velocity $(2 g R)^{1 / 2}$. Under conditions where this formulation is useful, the mean thermal speed is much smaller than the escape velocity, so the fraction of molecules moving fast enough to escape is small. It is also a strong function of molecular mass. Thus, on the inner planets today, Jeans escape is important only for hydrogen and helium. Heavier gases escape at negligibly small rates. In this section, I consider whether hydrodynamic escape also discriminates with respect to molecular mass.

Imagine an atmosphere composed largely of $\mathrm{H}_{2}$, which is flowing out into space with flux $F$, independent of altitude (neglecting the inverse square law dependence of flux on geocentric distance). The escaping hydrogen will tend to carry with it any minor atmospheric constituents with which it is mixed. If diffusion were negligible, the composition of the gas would be independent of altitude-mixing ratios of the minor gases would be constant. (The mixing ratio of constituent $i$ is the number density of $i$ divided by the total number density.) Diffusion, however, permits the heavier minor constituents to flow downwards, under the action of gravity, thereby in principle causing their preferential retention by the planet.

Whether this mass discrimination is important or not depends on the relative magnitudes of the hydrogen outflow velocity and the minor constituent diffusion velocity (Hunten, 1979; Sekiya et al., 1980).

For an isothermal atmosphere, the diffusion velocity, $w$, of a minor constituent relative to the ambient gas (see Walker, 1977) is

$w=-D[(1 / n)(\mathrm{d} n / \mathrm{d} z)+(m g / \mathrm{k} T)]$

where $n$ is the number density of the diffusing gas and $z$ is the altitude. The diffusion coefficient, $D$, is inversely proportional to ambient number density, say $D=\mathrm{b} / N$, where $\mathrm{b}$ is a constant. For constant mixing ratio, $[(1 / n)(\mathrm{d} n / \mathrm{d} z)]$ $-\overline{m g} / \mathrm{k} T$ where $\bar{m}$ is the mean molecular weight of the atmosphere, taken to be mostly $\mathrm{H}_{2}$. So, $w=-\mathrm{b} / N[(m-\bar{m}) \mathrm{g} / \mathrm{k} T]$. The minus sign indicates that diffusion is downwards for a gas with above-average molecular mass. Since ambient number density, $N$, decreases exponentially with height, the diffusion velocity, $w$, increases exponentially. But the outflow velocity, $w=F / N$ also increases exponentially with height. Whether the diffusion velocity is significant compared to the outflow velocity therefore depends on the relative magnitudes of $F$ and $[\mathrm{b}(m-\bar{m}) \mathrm{g} / \mathrm{k} T]$.

A representative value of $b$ is $10^{19} \mathrm{~cm}^{-1} \mathrm{~s}^{-1}$ (see Walker, 1977). For $\mathrm{N}_{2}$ in $\mathrm{H}_{2}$ at a temperature of $500 \mathrm{~K}$, I calculate $[\mathrm{b}(m-\bar{m}) \mathrm{g} / \mathrm{kT}]=6 \times 10^{12}$ $\mathrm{cm}^{-2} \mathrm{~s}^{-1}$. This is negligible compared with the estimated $\mathrm{H}_{2}$ escape flux of $2 \times 10^{14} \mathrm{~cm}^{-2} \mathrm{~s}^{-1}$. A higher temperature would be even more unfavorable to diffusion, but a heavier gas, such as krypton, might diffuse fast enough to be discriminated against by the escape process.

The conclusion is that hydrodynamic escape is unselective with respect to molecular mass provided the escape flux is considerably larger than $10^{13}$ $\mathrm{cm}^{-2} \mathrm{~s}^{-1}$. Smaller escape fluxes will definitely leave heavier gases behind. The escape flux implied by my interpretation of Ringwood's model is large in this sense. 


\section{CONDITIONS FOR THE ESCAPE OF LARGE QUANTITIES OF HYDROGEN}

For simplicity, I assume that the atmosphere is escaping into a nearvacuum, the nebula having dispersed at the time under consideration. The consequences of accretion in the presence of a gaseous nebula are explored in a later section.

Following Ringwood, I assume that the atmosphere is escaping while the Earth is accreting. Accretion is bringing to the Earth a mixture of hightemperature condensate rich in metallic iron and low-temperature condensate rich in water. Impact heating drives the water (and other volatiles) into the atmosphere where it reacts with excess iron, also heated by impact, to produce hydrogen and iron oxide. Under what circumstances will water remain in the atmosphere as steam rather than condensing on the surface?

The stability of a steam atmosphere against condensation has been examined by Ingersoll (1969). He finds that the key parameter is the flux of infrared energy leaving the top of the atmosphere. If this flux is too small, water vapor will condense. If it is sufficiently large, a steam atmosphere is stable. The critical flux is estimated to be about twice the present day flux of infrared radiation from the Earth, ca. $2.5 \times 10^{5} \mathrm{erg} \mathrm{cm}^{-2} \mathrm{~s}^{-1}$.

The rate of release of accretional energy may be estimated from the gravitational potential energy of the Earth. For a sphere of uniform density this energy is $3 / 5 M^{2} G / r_{o}=3 / 5 M g_{o} r_{o}=2.3 \times 10^{39} \mathrm{erg}$, where $G$ is the universal constant of gravitation, $M$ the mass, $r_{0}$ the radius and $g_{0}$ the gravitational acceleration at the surface. Division by the surface area of the Earth and a time for accretion $\tau$ s yields $F_{\mathrm{o}}=4.4 \times 10^{20} / \tau \mathrm{erg} \mathrm{cm}^{-2} \mathrm{~s}^{-1}$. For $\tau=10^{6} \mathrm{y}$, I calculate $F_{\mathrm{o}}=1.4 \times 10^{7} \mathrm{erg} \mathrm{cm}^{-2} \mathrm{~s}^{-1}$ and for $\tau=10^{8} \mathrm{y}$, $F_{\mathrm{o}}=1.4 \times 10^{5} \mathrm{erg} \mathrm{cm}^{-2} \mathrm{~s}^{-1}$. It seems likely that accretional heating in conjunction with solar luminosity would have prevented condensation of water vapor unless the accretion time exceeded $10^{8} \mathrm{y}$.

Any estimate of the composition of this transient atmosphere must of course be highly speculative. Using the values in Table I, assuming water reacts immediately, $\mathrm{C}$ is present as $\mathrm{CH}_{4}$, and $\mathrm{N}$ is present as $\mathrm{N}_{2}, \mathrm{I}$ calculate ratios of partial pressures, $\mathrm{H}_{2} / \mathrm{CH}_{4} / \mathrm{N}_{2}$ equal to $270 / 46 / 1$. The mean molecular mass of this atmosphere is ca. 4 amu.

The $\mathrm{H}_{2} / \mathrm{H}_{2} \mathrm{O}$ ratio would have depended on the rapidity of reaction between degassed water vapor and accreting iron. If conditions were such as to yield thermodynamic equilibrium at a temperature of $400^{\circ} \mathrm{C}$ the ratio would have been 130 (Ringwood, 1979), causing little change in mean molecular mass. A fairly large ratio is in any case implied by the model since water that is swept away to space is not available to oxidize iron, and a major reason for assuming a large initial volatile inventory was to provide oxygen in the form of water.

What can be said about the structure of an atmosphere composed mainly of hydrogen, with minor amounts of water and methane? In the lower atmosphere, presumably, heat would have been transported by convection 
and infrared radiation. Temperature would generally have declined with increasing altitude up to the level of radiative relaxation. Above this level the connection between temperature and the infrared radiation field would have been broken and heat transport would have been by conduction and advection. Radiative relaxation occurs when atmospheric density falls so low that energy-exchanging collisions between molecules are less frequent than emission of infrared photons. Under these conditions a molecule that is excited by absorption of an infrared photon loses its energy by reradiation rather than by collisional conversion to kinetic energy. On the Earth today, radiative relaxation occurs in the vicinity of the mesopause, where the ambient number density is ca. $10^{14} \mathrm{~cm}^{-3}$. It might be reasonable to suppose that radiative relaxation would have occurred at about the same number density in the primordial atmosphere, although the question certainly merits more careful study (the density could be much lower in a steam atmosphere).

The temperature at the level of radiative relaxation is related to the flux of infrared energy radiated by the atmosphere into space. It therefore depends on solar luminosity, planetary albedo, and the rate of accretional heating. A temperature near $300 \mathrm{~K}$ would seem reasonable. Perhaps local heating by planetesimal bombardment in the vicinity of the mesopause would raise the temperature somewhat. In what follows I shall use a generous estimate for this temperature of $500 \mathrm{~K}$.

The height of the level of radiative relaxation depends on atmospheric mass and temperatures in the lower atmosphere. Atmospheric mass depends on the relative rates of escape and of the accretional source of atmospheric gas. There seems to be no way to estimate these relative rates at this time. I shall simply assume that the height of the relaxation level was much less than the radius of the Earth so that gravitational acceleration at this level was approximately equal to its surface value.

How rapidly can this atmosphere escape? The escape flux is limited by the rate at which energy can be supplied to lift the gas against the force of gravity. Below the level of radiative relaxation there should be no problem. The flux of infrared energy is more than enough to sustain the rates of outflow of hydrogen under discussion. The problem arises in the thermosphere, above the level of radiative relaxation. Energy is in short supply in the thermosphere. The heat flux provided to the thermosphere today by solar ultraviolet radiation is only about $1 \mathrm{erg} \mathrm{cm}^{-2} \mathrm{~s}^{-1}$ (Torr et al., 1980). If all of this energy were used to carry atmospheric gas out of the Earth's gravitational potential well the rate of outflow would be $F=1 / \bar{m} r_{o} g=2 \times 10^{11}$ $\mathrm{cm}^{-2} \mathrm{~s}^{-1}$ for $\vec{m}=4$ amu. Evidently a much larger thermospheric heat source is required to sustain an escape flux of $2 \times 10^{14} \mathrm{~cm}^{-2} \mathrm{~s}^{-1}$. (Today, of course, most of the thermospheric heat is removed by conduction downwards to the mesopause, not by evaporation of gas to space.)

In order to clarify the energetic limitations on the escape process assume for the moment that there is no thermospheric heat source. Assume, 
further, that thermal conduction is negligible, so that only the intrinsic thermal energy of the gas is available to raise it out of the Earth's gravitational potential well. Allowing for spherical geometry, the outward flux at level $r$ is $F(r)=F_{\mathrm{o}}\left(r_{\mathrm{o}} / r\right)^{2} \mathrm{~cm}^{-2} \mathrm{~s}^{-1}$. The thermal energy content of the gas (assumed to be either monatomic or $\mathrm{H}_{2}$ ) flowing upwards through level $r$ is $F 5 / 2 \mathrm{kT}(r)$ erg cm $\mathrm{cm}^{-2} \mathrm{~s}^{-1}$. The energy required to carry this gas to infinite distance is $M m G F / r \mathrm{erg} \mathrm{cm}^{-2} \mathrm{~s}^{-1}$ where $M$ is the mass of the Earth, $m$ the molecular mass of the gas, and $G$ the universal constant of gravitation. Equating the advected energy flux to the escape energy flux

$F \mathrm{5} / 2 \mathrm{k}\left(T-T_{\infty}\right)=F M m G / r$

or

$T(r)=T_{\infty}+2 / 5(M m G / \mathrm{kr})$

where $T_{\infty}$ is the temperature of the gas at infinite distance. This temperature cannot be less than $0 \mathrm{~K}$, so $T(r)>2 M m G / 5 \mathrm{kr}$, and $T_{\mathrm{o}}>2 M m G / 5 \mathrm{kr} r_{\mathrm{o}}$ $=2 / 5\left(m g r_{0} / \mathrm{k}\right)$, where $T_{\mathrm{o}}$ is the temperature at the base of the thermosphere. Escape under the circumstances described is possible only if $1>$ $2 / 5\left(m g r_{o} / k T_{o}\right)=2 / 5\left(r_{o} / H_{o}\right)$. (Thermal energy exceeds gravitational potential energy.) For $\mathrm{H}_{2}$ in the Earth's gravitational field this condition is met only if $T_{\mathrm{o}}>6000 \mathrm{~K}$. There is very little likelihood of a temperature this high at the base of the thermosphere near the level of radiative relaxation.

But the assumed conditions were unrealistic. Under most circumstances conduction of heat will be more important than advection. The conducted heat flux is $-K(\mathrm{~d} T / \mathrm{d} r)$ where $K$ is the thermal conductivity. If the heat flux is equated to the flux of escaping energy and $T_{\infty}$ is assumed to be zero, a differential equation for $T(r)$ results in

$\mathrm{d} T / \mathrm{d} r=-\left(F_{\mathrm{o}} M m G r_{\mathrm{o}}{ }^{2}\right) /\left(K r^{3}\right)$

which has as its solution

$T(r)=T_{\mathrm{o}}+\left(F_{\mathrm{o}} M m G / 2 K\right)\left[\left(r_{\mathrm{o}} / r\right)^{2}-1\right]$

Clearly, $F_{\mathrm{o}}$ cannot be greater than $2 K T_{\mathrm{o}} / M m G$. If it were, temperature would be negative at great heights. For molecular hydrogen, $K=2 \times 10^{5}$ erg cm $\mathrm{cm}^{-1} \mathrm{~s}^{-1} \mathrm{deg}^{-1}$, and I calculate $F_{\mathrm{o}}<1.5 \times 10^{11} \mathrm{~cm}^{-2} \mathrm{~s}^{-1}$ for $T_{\mathrm{o}}=$ $500 \mathrm{~K}$. The inclusion of advective energy in the energy balance would raise this limit, but by very little.

Note that this appealingly simple result is not a solution of the escape problem. The flux may be smaller than the calculated limit, which would yield non-zero temperature at infinite distance. A solution for the escape flux requires consideration of the momentum balance and continuity equations as well as the energy balance equation (Parker, 1958, 1964, 1971; Banks and Holzer, 1968, 1969a, 1969b; Yeh, 1970; Holzer et al., 1971; 
Strobel and Weber, 1972). But, for present purposes, the upper limit is sufficient. An escape flux of $2 \times 10^{14} \mathrm{~mol} \mathrm{~cm}^{-2} \mathrm{~s}^{-1}$ is not possible in the absence of thermospheric heat sources.

The escape of a hydrogen atmosphere in the presence of thermospheric heating has been considered by Watson et al. (1981). They have shown that the fate of the energy deposited in the thermosphere depends on the height above the level of radiative relaxation at which it is deposited. If the level of heating is fairly close to the relaxation level, temperature increases from this level to a maximum at the level of heat deposition and then decreases monotonically out to space. The energy deposited in the thermosphere is removed partly by conduction down to the relaxation level and partly by advection and conduction out to space. The escape energy flux is considerably less than the energy deposited in the thermosphere.

If, on the other hand, thermospheric heat is deposited well above the level of radiative relaxation, temperature declines to a minimum between this level and the level of heating. This temperature decrease is a consequence of cooling by expansion as in the case already considered of no thermospheric heat source. Temperature rises to a maximum at the level of heat input and then decreases monotonically to space. In this situation the conductive heat flow is upward at the relaxation level, so all of the energy deposited in the thermosphere must be removed by escape. The escape energy flux is then equal to the thermospheric heat source augmented by the heat flux conducted upwards from below. I have already shown that the heat flux from below can contribute at most about $10^{11} \mathrm{~cm}^{-2} \mathrm{~s}^{-1}$ to the escape flux. The energy to sustain an escape flux of $2 \times 10^{14} \mathrm{~cm}^{-2} \mathrm{~s}^{-1}$ must therefore be provided almost entirely by thermospheric heating. The heat source required is $875 \mathrm{erg} \mathrm{cm}^{-2} \mathrm{~s}^{-1}$ for a mean molecular mass of 4 . The present-day thermospheric heat source is about $1 \mathrm{erg} \mathrm{cm}^{-2} \mathrm{~s}^{-1}$.

Where could all this thermospheric energy have come from? Presumably, either from greatly enhanced solar luminosity in the extreme ultraviolet or else from a greatly enhanced solar wind, or a combination of the two. The solar wind source may be less plausible because early core formation combined with rapid internal convection may have generated a large geomagnetic field. Accretional energy is not likely to have contributed to thermospheric heating because even quite small planetesimals deposit their energy at or below the level of radiative relaxation.

The height above the relaxation level of either the ultraviolet or solar wind heat sources depends on the density at this level and the overlying temperature profile. Watson et al. (1981) have shown that a hydrogen density of $10^{14} \mathrm{~cm}^{-3}$ at the relaxation level leads to a well-developed temperature minimum in the thermosphere. Under these circumstances, the escape energy flux would have been nearly equal to the thermospheric heating rate.

I conclude that the large escape flux implied by Ringwood's (1979) model is possible, but only if the thermospheric heat source was a thousand times larger than it is today. The Sun might have passed through a phase of 
high UV luminosity on its approach to the main sequence, or the energy might have been provided by a T-Tauri solar wind (Strom, 1977; Gehrels. 1978; Zahnle and Walker, 1982).

The photochemistry of a massive hydrogen thermosphere in the presence of large fluxes of ionizing radiation and rapid outflow merits investigation. I shall simply assume, for purposes of discussion, that $\mathrm{H}_{2}$ remains the dominant species. If $\mathrm{H}$ were more abundant escape would be easier, but the energy requirement would be greater (because energy is needed to dissociate $\mathrm{H}_{2}$ ). If the gas were fully-ionized, escape would be further facilitated by a lowering of mean molecular weight, but electromagnetic interactions would introduce complications.

The ultimate fate of this presumed secondary atmosphere depends on the relative time scales of accretion and enhanced thermospheric heating. If accretion tapered off before the thermospheric energy source the atmosphere could have dissipated completely. The present atmosphere would then be a product of later degassing. If the energy source tapered off before the atmosphere had completely escaped, however, the present atmosphere could be a residual. As the energy source decreased the escape flux would decrease also, until the heaviest gases began to be preferentially retained. Decreasing escape flux would lead to retention of progressively lighter gases. A residual atmosphere could therefore be enriched in heavy gases. The extent of the enrichment would depend on the time scale associated with the decrease of the thermospheric energy source.

A multitude of possible scenarios can be imagined. It does not seem possible to choose among them without a lot more study.

\section{EFFECTS OF ROTATION}

Ringwood (1979) has advanced arguments that the initial rotation period of the Earth was as short as $5 \mathrm{~h}$ and has suggested that escape of a massive atmosphere could have been facilitated by rotational energy. How important is rotation likely to have been? I assume that the lower atmosphere was constrained by eddy viscosity and free convection to rotate with the Earth. At the level of radiative relaxation, the effective gravitational acceleration was reduced by the centripetal acceleration, $\Omega^{2} r$. For a rotational period of $5 \mathrm{~h}, \Omega=3.5 \times 10^{-4} \mathrm{rad} \mathrm{s} \mathrm{s}^{-1}$. The centripetal acceleration is therefore only about $80 \mathrm{~cm} \mathrm{~s}^{-2}$ for $r$ approximately equal to the radius of the Earth. At the present level of approximation this correction can be ignored.

Assume that viscosity was negligible above the level of radiative relaxation, i.e. the height profile of horizontal velocity was dominated by conservation of the angular momentum of the outflowing gas. The angular velocity of this gas at the equator was then $\omega=\Omega\left(r_{0} / r\right)^{2}$. The effective gravitational acceleration in the thermosphere would be

$g^{\prime}=g-\omega^{2} r=g_{o}\left(r_{o} / r\right)^{2}-\Omega^{2}\left(r_{o} / r\right)^{4} r$

so the relative importance of centripetal acceleration decreases with increasing altitude. 
The possible role of viscosity could be explored by comparing the escape flux of angular momentum with the viscous flux. For the large escape fluxes of interest molecular viscosity is almost certainly negligible. I shall now argue that the thermosphere was stably stratified and non-turbulent so that eddy viscosity was not a factor. The critical parameter is the Richardson number

$R i=\omega_{\mathrm{B}}{ }^{2} /\left(U^{\prime}\right)^{2}$

where $\omega_{B}$ is the frequency of vertical buoyancy oscillations of the atmosphere (the Brunt-Vaisala frequency), and $U^{\prime}$ is the vertical gradient in horizontal wind (Hess, 1959).

$\omega_{\mathrm{B}}^{2}=g / T\left(\Gamma_{\mathrm{a}}-\Gamma\right)$

where $\Gamma_{\mathrm{a}}$ is the adiabatic temperature lapse rate and $\Gamma$ is the ambient lapse rate. Conduction of heat in the escaping gas makes $\Gamma \ll \Gamma_{\mathrm{a}}=0.7 \mathrm{~K} \mathrm{~km} \mathrm{~km}^{-1}$ for $\mathrm{H}_{2}$, so $\omega_{\mathrm{B}}^{2}=10^{-5} \mathrm{~s}^{-2}$ for $T=500 \mathrm{~K}$.

The horizontal wind speed

$$
\begin{aligned}
U & =\omega r \\
& =\Omega r_{\mathrm{o}}\left(r_{\mathrm{o}} / r\right)
\end{aligned}
$$

under conditions where angular momentum is conserved The square of the wind shear is therefore $\left(U^{\prime}\right)^{2}=\omega^{2}=10^{-7}$ at most, and the Richardson number, $R i \geqslant 100$. The atmosphere is stable against mechanical turbulence for $R i>0.25$. When the escape flux is large, horizontal wind speeds in the thermosphere are dominated by conservation of angular momentum and the horizontal flow is laminar. The decrease in $g$ with altitude is offset by the decrease in $U^{\prime}$ with altitude.

The viscosity of $\mathrm{H}_{2}$ at $500 \mathrm{~K}$ is $\eta=167 \times 10^{-6} \mathrm{~g} \mathrm{~cm}^{-1} \mathrm{~s}^{-1}$ (Weast, 1978). For $U^{\prime}=\omega<3.5 \times 10^{-4} \mathrm{~s}^{-1}$ the vertical flux of horizontal momentum transported by viscosity is $\eta U^{\prime}<5.8 \times 10^{-8} \mathrm{~g} \mathrm{~cm}^{-1} \mathrm{~s}^{-2}$. The advected vertical flux of horizontal momentum is $F \bar{m} \Omega r_{0}=3 \times 10^{-4}$ $\mathrm{g} \mathrm{cm}^{-1} \mathrm{~s}^{-2}$ for $F=2 \times 10^{14} \mathrm{~cm}^{-2} \mathrm{~s}^{-1}$ and $\bar{m}=4$ amu. For large escape fluxes, therefore, viscosity has negligible affect on the velocity profile in the thermosphere.

\section{ACCRETION IN THE PRESENCE OF A GASEOUS NEBULA}

In all of the above I have assumed that the Earth and its atmosphere were surrounded by the vacuum of interplanetary space. If accretion was rapid, however, it is perhaps more likely that the Earth accreted in the interior of a gaseous nebula. The presence of such a nebula would have had a profound effect on the Earth's earliest atmosphere (Hayashi et al., 1979). In exploring this effect I shall, for simplicity, assume that the Earth achieved its present mass before the nebula dispersed. The arguments and 
calculations can be readily adjusted to accommodate an Earth only partially formed at the time of disappearance of the nebula.

I shall consider first the pressure profile within an atmosphere of gravitationally concentrated nebular gas and shall set a lower limit on the temperature of this gas. Next, I shall argue that much of the atmosphere must have been in motion in order to inhibit diffusive separation of the heavier constituents of the gas mixture. Consideration of the temperature profile in a freely convecting atmosphere suggests that convection was limited to lower altitudes. Estimates of energy transport by radiation and convection set some limits on the thickness of the convective layer and confirm the earlier lower limit on the temperature of the nebular gas.

The nebular gas is concentrated by the Earth's gravitational field to form a primary atmosphere. If this atmosphere is isothermal, the variation of pressure with geocentric distance is readily calculated by equating pressure gradient to gravitational acceleration (Walker, 1977).

$p(r)=p_{\infty} \exp \left(r_{\mathrm{o}}^{2} / r H_{\mathrm{o}}\right)$

where $p_{\infty}$ is the pressure of the nebula at infinite distance from the Earth, $r_{\mathrm{o}}$ is a reference level which might be the Earth's surface, and $H_{\mathrm{o}}=\mathrm{kT} / \mathrm{mg}\left(r_{\mathrm{o}}\right)$. The mass of the atmosphere has been assumed negligible compared with the mass of the Earth.

The pressure at the surface is

$p_{\mathrm{o}}=p_{\infty} \exp \left(r_{\mathrm{o}} / H_{\mathrm{o}}\right)$

If the atmosphere was composed of $\mathrm{H}_{2}$ at a temperature of $1500 \mathrm{~K}$, I find $H_{\mathrm{o}}=636 \mathrm{~km}$. A nebular pressure of $10^{-4} \mathrm{bar}$ is often assumed (Cameron and Pine, 1973; Larimer, 1973; Hunten, 1979; Hayashi et al., 1979). So $p_{\mathrm{o}}=2.2 \mathrm{bar}=2.3 \times 10^{6} \mathrm{dyn} \mathrm{cm}^{-2}$. The mass per unit area of the atmosphere is $p_{\mathrm{o}} / \mathrm{g}=2.3 \times 10^{3} \mathrm{~g} \mathrm{~cm}^{-2}$ so the total mass of the atmosphere is $1.2 \times 10^{22} \mathrm{~g}$, which is negligible compared with the mass of the Earth $\left(6 \times 10^{27} \mathrm{~g}\right)$.

But suppose the temperature was less, say $500 \mathrm{~K}$. Then, $H_{\mathrm{o}}=212 \mathrm{~km}$, $p_{\mathrm{o}} / p_{\infty}=\exp (30)=2.0 \times 10^{13}, p_{\mathrm{o}}=2 \times 10^{9} \mathrm{bar}$, and the mass of the atmosphere is $10^{31} \mathrm{~g}$. Obviously, the mass of the gravitationallyconcentrated primary atmosphere is a very strong function of temperature and mean molecular mass.

An atmospheric mass comparable to the Earth's mass would increase gravitational acceleration and lead to further concentration of gas. In fact, the atmosphere would grow without limit as long as the supply of nebular gas was not exhausted. It is presumably just this gravitational instability that produced the massive hydrogen atmospheres of the outer planets (Mizuno et al., 1978). The absence of such atmospheres on the inner planets provides a lower limit on the temperature of the nebula in which these planets accreted. I shall now estimate this limit for the Earth. 
I require that the mass of the atmosphere be much less than the mass of the Earth or $p_{\mathrm{o}} \ll M_{\mathrm{e}} g / S_{\mathrm{e}}$, where $M_{\mathrm{e}}$ is the mass of the Earth, $S_{\mathrm{e}}$ is its surface area, and $g$ is gravitational acceleration. The limit is $p_{0} \ll 10^{12}$ dyn $\mathrm{cm}^{-2} \simeq 10^{6}$ bar. Now, $p_{\mathrm{o}} / p_{\infty}=\exp \left(r_{\mathrm{o}} / H_{\mathrm{o}}\right)$, so for $p_{\infty}=10^{-4}$ bar I require $r_{\mathrm{o}} / H_{\mathrm{o}} \ll 23$. This expression yields $H_{\mathrm{o}}>277 \mathrm{~km}$ or $T>650 \mathrm{~K}$. A gas of higher molecular weight than $\mathrm{H}_{2}$ would require a correspondingly higher temperature.

If the atmosphere were stationary, neither expanding nor contracting nor in turbulent motion, it could be expected to have the same temperature as the surrounding nebular gas over much of its extent. The temperature might increase near the surface as a result of accretional heating. Direct solar heating would presumably be unimportant because of the opacity of the nebula. A high temperature for the nebular gas around the Earth would not conflict with accretion of low temperature condensates. As Ringwood has suggested, these could condense in the cool outer regions of the nebula, well above the ecliptic plane, and then settle through the hotter gas near the ecliptic plane to become available for accretion.

If the atmosphere were static, however, its different constituents could diffuse relative to one another and the heavier constituents could suffer much greater gravitational concentration than hydrogen. For ${ }^{36} \mathrm{Ar}$, for example, the scale height is $35.5 \mathrm{~km}$ at a temperature of $1500 \mathrm{~K}$, and the surface pressure is $\mathrm{e}^{180}=10^{78}$ times the pressure in the nebula. This result is plainly unacceptable. Diffusive separation must be inhibited in the primary atmosphere by mass motion, presumably turbulence, or else the temperature must be very much larger than $1500 \mathrm{~K}$, or both. Alternatively, perhaps the lifetime of the gaseous nebula was too short to permit minor gases to achieve diffusive equilibrium in the primary atmosphere.

A heuristic derivation of the characteristic time for establishment of diffusive equilibrium appears in Walker (1977). This time is

$\tau_{\mathrm{D}}=2 \sigma v H N / g$

where $\sigma$ is the collision cross section, $v$ the mean thermal speed of the diffusing molecules, $H=\mathrm{k} T / \bar{m} g$ the ambient atmospheric scale height, $N$ the ambient number density, and $g$ the gravitational acceleration. For an isothermal atmosphere with the pressure profile given by eq. 10 , this expression becomes

$\tau_{\mathrm{D}}=\left(2 \sigma u p_{\infty} / \bar{m} g_{\mathrm{o}}^{2}\right)\left(r^{2} / r_{\mathrm{o}}^{2}\right) \mathrm{e}^{\left(r_{\mathrm{o}}^{2} / r H_{\mathrm{o}}\right)}$

where $g_{0}$ is the gravitational acceleration and $H_{0}$ is the scale height at the ground, $r_{0}$, and $p_{\infty}$ is the nebular pressure.

The diffusion time is a minimum at $r / r_{0}=r_{0} / 2 H_{0} \simeq 5$ for an atmosphere of $\mathrm{H}_{2}$ at $1500 \mathrm{~K}$. At this level the diffusion time for ${ }^{36} \mathrm{~A}$ is only $35 \times 10^{3} \mathrm{y}$, for $p_{\infty}=10^{-4}$ bar. At the ground it is $5 \times 10^{6} \mathrm{y}$. These times are fairly short. It seems likely that mass motion and mixing of the atmosphere must be invoked to inhibit diffusive separation. 
Accretional heating might have driven free convection in the lower portions of the atmosphere. In the upper levels, where the infrared opacity was presumably low, turbulence, if present, was most probably generated by wind shear. The source of mechanical energy was presumably rotation of the Earth relative to the surrounding nebular gas. How plausible is a fully turbulent primary atmosphere?

If the atmosphere was strongly turbulent at all levels it would not have been isothermal. Instead, the temperature lapse rate might have approximated the adiabatic lapse rate, $g / c_{p}$.

$\mathrm{d} T / \mathrm{d} r=-\mathrm{g} / c_{\mathrm{p}}=-\mathrm{g}_{\mathrm{o}} / c_{\mathrm{p}}\left(r_{\mathrm{o}} / r\right)^{2}$

Therefore

$T(r)=T_{\infty}+\left(g_{o} r_{o}^{2} / c_{p} r\right)$

Using $c_{\mathrm{p}}=9 / 2(\mathrm{k} / \mathrm{m})$ appropriate to a diatomic gas at high temperature, I calculate $T_{\mathrm{o}}=T_{\infty}+3400 \mathrm{~K}$. The temperature near the ground in an adiabatic atmosphere is therefore high enough to evaporate most terrestrial materials. Such a result is unacceptable. Evidently, the lapse rate must have been subadiabatic somewhere, probably at higher levels where low densities yielded an atmosphere relatively transparent to infrared radiation.

As an approximation to the temperature profile in a radiative-convective primary atmosphere, suppose that the atmosphere was isothermal above $r_{\mathbf{m}}$ and adiabatic below this height. Then

$T(r)=T_{\infty}+\left(g_{\mathrm{o}} r_{\mathrm{o}} / c_{\mathrm{p}}\right)\left(r_{\mathrm{o}} / r-r_{\mathrm{o}} / r_{\mathrm{m}}\right), \quad r<r_{\mathrm{m}}$

If I assume $T\left(r_{\mathrm{o}}\right)<T_{\infty}+1700 \mathrm{~K}$, which might be a reasonable upper limit, I require $r_{\mathrm{m}} / r_{\mathrm{o}}<2$.

A lower limit for the pressure at the top of the adiabatic region follows from (10) for the pressure profile in an isothermal atmosphere. For $T_{\infty}=$ $500 \mathrm{~K}$ I calculate $p\left(r_{\mathrm{m}}\right)>335 \mathrm{bar}$ and for $T_{\infty}=1000 \mathrm{~K}, p\left(r_{\mathrm{m}}\right)>0.18 \mathrm{bar}$, where $p_{\infty}=10^{-4}$ bar is assumed. I now consider whether these pressures are consistent with the absence of free convection in the overlying atmosphere.

The flux of energy carried by radiation in a grey atmosphere, according to the Eddington approximation (Goody, 1964), is

$F=-\left(16 \sigma T^{3} / 3 \kappa \rho\right)(\mathrm{d} T / \mathrm{d} r)$

where $\sigma$ is the Stefan-Boltzmann constant, $\kappa \mathrm{cm}^{2} \mathrm{~g}^{-1}$ the opacity of the atmosphere, and $\rho=m p / k T$ the mass density. If the adiabatic lapse rate $-\mathrm{d} T / \mathrm{d} r=g / c_{\mathrm{p}}$ is substituted into this expression there results a lower limit on the outward flux of energy in the adiabatic region of the atmosphere; heat transport by convection is additional to that by radiation.

$F=16 \sigma T^{4} g / 10.5 \kappa \rho$ 
The energy source in the lower atmosphere provided, presumably, by accretion must be at least this large in order to sustain free convection. At the top of the convective layer

$F\left(r_{\mathrm{m}}\right)=F_{\mathrm{o}}\left(r_{\mathrm{o}} / r_{\mathrm{m}}\right)^{2}=16 \sigma T_{\infty}^{4} g_{\mathrm{o}} / 10.5 \kappa \rho\left(r_{\mathrm{m}}\right)\left(r_{\mathrm{o}} / r_{\mathrm{m}}\right)^{2}$

or

$F_{\mathrm{o}} \kappa=16 \sigma T_{\infty}^{4} g_{\mathrm{o}} / 10.5 \rho\left(r_{\mathrm{m}}\right)$

where $F_{0}$ is the energy source at the bottom of the atmosphere. Using the lower limits already calculated for $p\left(r_{\mathrm{m}}\right)$ I find $F_{\mathrm{o}} \kappa<15.8 \mathrm{erg} \mathrm{g}^{-1} \mathrm{~s}^{-1}$ for $T_{\infty}=500 \mathrm{~K}$ and $F_{\mathrm{o}^{K}}<4.7 \times 10^{5} \mathrm{erg} \mathrm{g}^{-1} \mathrm{~s}^{-1}$ for $T_{\infty}=1000 \mathrm{~K}$. If $F_{\mathrm{o}^{K}}$ were to exceed these limits then $r_{\mathrm{m}} / r_{\mathrm{o}}$ would exceed 2 and unreasonably large surface temperatures would be implied.

The energy flux due to accretion was estimated earlier from the total gravitational potential energy of the Earth as $F_{\mathrm{o}}=4.4 \times 10^{20} / \tau \mathrm{erg} \mathrm{cm}^{-2} \mathrm{~s}^{-1}$, where $\tau$ is the accretion time. For $\tau=10^{7} \mathrm{y}=3.1 \times 10^{14} \mathrm{~s}$, for example, we have $F_{0}=1.4 \times 10^{6} \mathrm{erg} \mathrm{cm}^{-2} \mathrm{~s}^{-1}$, nearly equal to the solar constant. The limits on $F_{\mathrm{o}^{\kappa}}$ derived above then require $\kappa<1.1 \times 10^{-5} \mathrm{~cm}^{2} \mathrm{~g}^{-1}$ for $T_{\infty}=500 \mathrm{~K}$ and $\kappa<0.34 \mathrm{~cm}^{2} \mathrm{~g}^{-1}$ for $T_{\infty}=1000 \mathrm{~K}$. Mizuno et al. (1978, 1980 ) suggest that opacities in the primary atmosphere, with allowance for dust, may have been between $10^{-4}$ and $1 \mathrm{~cm}^{2} \mathrm{~g}^{-1}$. The opacity limit calculated for $T_{\infty}=500 \mathrm{~K}$ seems impossibly low. Unless the accretion time was about $10^{8}$ years or longer it seems that the nebular gas near the Earth must have been hotter than $500 \mathrm{~K}$. A low nebular temperature yields a dense primordial atmosphere, opaque to infrared radiation. Accretional heating leads to a thick convective layer with an adiabatic temperature profile and a high surface temperature.

No obvious thermal inconsistencies emerge for the case of $1000 \mathrm{~K}$ nebular temperature. Depending on opacity and accretion rate, the convective layer could have been less than one Earth radius in thickness and the surface temperature less than $2700 \mathrm{~K}$. A relatively thin convective layer, however, does not solve the problem of gravitational concentration of heavy gases under the action of diffusion. At altitudes above $r_{m}$ the atmosphere must have been mixed by processes other than free convection. The likeliest candidate is mass motion driven by the Earth's rotation.

\section{CIRCULATION OF THE PRIMARY ATMOSPHERE}

I do not know of any general treatment of the circulation of the atmosphere of a rotating planet immersed in a nebula, but the qualitative features of this circulation can be sketched. A numerical solution is available for the related problem of a rotating disk in an infinite, incompressible, viscous fluid (Schlichting, 1955). The fluid flows toward the disk along the axis of rotation, acquires angular velocity from the disk, and flows outward 
in the plane of the disk under the action of the centrifugal force. The motion of an incompressible fluid around a rotating sphere should be much the same, with downward flow over the poles and outward flow over the equator. Such motion could, in principle, eliminate anomalies in the composition of the primary atmosphere.

The compressibility of a planetary atmosphere introduces complications however. If the temperature profiles were adiabatic everywhere these complications might be minor, but I have argued above for a subadiabatic temperature lapse rate at high altitudes. A subadiabatic lapse rate corresponds to stable stratification, in which vertical motions are resisted by buoyant forces. The overturning circulation would have to work against buoyancy as well as against viscous forces. Buoyant stability loses its force when the thermal relaxation time of the fluid is short compared with the time scale for temperature change caused by vertical motion and compression. This condition is most likely to be satisfied at high altitudes where densities and heat capacities are low. More study will be needed to decide whether the entire atmosphere can participate in the overturning motion or whether this circulation is restricted to the outer levels, with the underlying atmosphere in almost rigid rotation.

In the rest of this section I shall attempt a qualitative description of the circulation, starting with the simple, analytical solution for the case of slow rotation. I shall consider the modifications to this solution likely to result from fast rotation and then the possible impact of free convection in the lower atmosphere. The discussion will suggest that rotation does not markedly reduce the gravitational binding of the atmosphere. I shall then argue that the circulation is not likely to generate mechanical turbulence in the upper atmosphere; turbulence would invalidate my assumption of nearly isothermal conditions in this region. I shall conclude that the hypothesis that atmospheric circulation driven by planetary rotation maintained a homogeneous primary atmosphere of nebular composition is not contradicted by obvious principles of atmospheric physics.

The motion of a viscous, incompressible fluid satisfies the Navier-Stokes equation (Landau and Lifschitz, 1959)

$$
\partial \mathrm{V} / \partial t+(\mathrm{V} . \nabla) \mathrm{V}=-1 / \rho \nabla p+\eta / \rho \nabla^{2} \mathrm{~V}
$$

where $\eta$ is the dynamic viscosity, assumed constant. If the motion of the fluid about a rotating sphere is assumed to be entirely zonal (azimuthal) as well as steady and symmetrical about the axis of rotation this equation reduces to

$$
\nabla^{2} V_{\phi}=0
$$

with solution in spherical coordinates

$$
\begin{aligned}
& \mathrm{V}_{\phi}=\left(\mathrm{ar}+\mathrm{b} / \mathrm{r}^{2}\right) \sin \theta \\
& \text { or angular velocity } \omega=\mathrm{V}_{\phi} / r \sin \theta=\mathrm{a}+\mathrm{b} / \mathrm{r}^{3}
\end{aligned}
$$


where $\Omega=a+b / r_{o}{ }^{3}$ is the angular velocity of the sphere of radius $r_{\mathrm{o}}$ (Lamb, 1932). The first term corresponds to rigid rotation of the fluid. It is zero if the fluid at great distance from the sphere is at rest.

This solution is correct only for small values of $\Omega r_{0}{ }^{2} \rho / \eta$. The rotation must be so slow and the fluid so viscous that perturbations of the pressure distribution are negligible. If the centrifugal force is important, pressure decreases less rapidly with radius over the equator than over the poles. The resulting horizontal pressure gradient causes equatorward motion near the sphere and poleward motion at great distances, with downwelling over the poles and upwelling over the equator. The interaction of the Coriolis force with these vertical and meridional motions will tend to reduce the angular velocity of the fluid at the equator and increase it at high latitudes, but the flow is still likely to be largely zonal. The solution in eq. 23 may approximate the globally-averaged angular velocity, yielding an overestimate at low latitudes and an underestimate at high latitudes.

Then, for an effective eddy viscosity independent of radius

$\omega=\Omega\left(r_{\mathrm{o}} / r\right)^{3}$

In fact, we might expect the eddy viscosity to be markedly higher in the freely convecting lower atmosphere than in the overlying region presumed to exhibit a subadiabatic temperature lapse rate. In this case the solution in eq. 23 would apply separately to the two regions, with the constants $a$ and $b$ chosen to satisfy the boundary conditions $\left(\omega=\Omega\right.$ at $r=r_{0}, \omega=0$ at $\left.r=\infty\right)$ and to provide a continuous outward flux of angular momentum. If the difference in viscosity between upper and lower regions were large, the lower region could be expected to rotate almost rigidly with the planet, while eq. 24 would apply to the upper region with $r_{\mathrm{o}}$ replaced by $r_{\mathrm{m}}$, the radius of the change in viscosity.

The effective gravitational acceleration at high altitudes over the equator would then be

$g^{\prime}=g_{\mathrm{o}}\left(r_{\mathrm{o}} / r\right)^{2}-\Omega^{2} r_{\mathrm{m}}\left(r_{\mathrm{m}} / r\right)^{5}$

For a rotational period of $5 \mathrm{~h}$ for the planet and for $r_{\mathrm{m}} / r_{\mathrm{o}}<2$ this expression is always positive, with a minimum value at $r=r_{\mathbf{m}}$.

The stability of the upper level flow against mechanical turbulence can be examined by calculating the Richardson number. For $r=r_{m}=2 r_{0}$ we have $g^{\prime}=125 \mathrm{~cm} \mathrm{~s}^{-2}$ and $\Gamma_{\mathrm{a}}=g^{\prime} / c_{\mathrm{p}}=0.08 \mathrm{~K} \mathrm{~km}^{-1}$, with $c_{\mathrm{p}}=1.5 \times 10^{8}$ erg $\mathrm{g}^{-1} \mathrm{deg}^{-1}$ for $\mathrm{H}_{2}$ at room temperature. The square of the buoyancy frequency in an isothermal atmosphere is $\omega_{\mathrm{B}}{ }^{2}=\left(g^{\prime} / T\right) \Gamma_{\mathrm{a}}=10^{-7} \mathrm{~s}^{-2}$ for $T=$ $1000 \mathrm{~K}$. The horizontal wind speed

$U=\omega r=\Omega r_{\mathrm{m}}\left(r_{\mathrm{m}} / r\right)^{2}$

so $U^{\prime}=2 \omega=7 \times 10^{-4}$ at most. So $R i=\left(\omega_{\mathrm{B}} / U^{\prime}\right)^{2}>0.2$ and the flow is marginally unstable against mechanical turbulence for the worst case assump- 
tion of $r=r_{m}=2 r_{0}$. Calculation of the Reynolds number (Hess, 1959) indicates that viscous damping is negligible. Stability increases rapidly for larger $r$ or smaller $r_{m}$, so throughout most of the thermosphere the flow would be laminar. There are therefore no obvious defects with the picture of a freely convecting, rigidly rotating, approximately adiabatic lower atmosphere overlain by a non-turbulent, approximately isothermal upper atmosphere exhibiting a global circulation driven by planetary rotation.

Convection below and circulation above may have prevented the composition of the primary atmosphere from departing significantly from that of the nebula.

Suppose, now, that massive degassing occurred during the course of accretion. The added gases might have been diluted by nebular hydrogen and dispersed into the nebula by convection and circulation without presenting any particular problem of escape. Such a model has been proposed by Ringwood (1979).

It remains to calculate the pressure profile in the adiabatic region of the atmosphere and to estimate the surface pressure. In hydrostatic balance

$\mathrm{d} p / \mathrm{d} r=-\rho g=-p(m g / \mathrm{k} T)$

Therefore

$$
\begin{aligned}
\frac{1}{p} \frac{\mathrm{d} p}{\mathrm{~d} r} & =-m g_{\mathrm{o}} r_{\mathrm{o}}{ }^{2} / \mathrm{kr}^{2}\left(T_{\infty}-\frac{g_{\mathrm{o}} r_{\mathrm{o}}^{2}}{c_{\mathrm{p}} r_{\mathrm{m}}}+\frac{g_{\mathrm{o}} r_{\mathrm{o}}{ }^{2}}{c_{\mathrm{p}} r}\right) \\
& =-1 / r\left[\frac{\mathrm{k}}{c_{\mathrm{p}} m}+\left(\frac{\mathrm{k} T_{\infty}}{m g_{\mathrm{o}} r_{\mathrm{o}}{ }^{2}}-\frac{\mathrm{k}}{c_{\mathrm{p}} m r_{\mathrm{m}}}\right) r\right]
\end{aligned}
$$

Integration yields

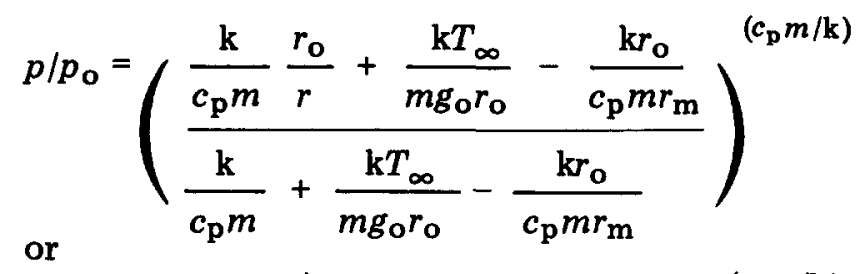

$p\left(r_{\mathrm{m}}\right) / p_{\mathrm{o}}=\left[1 / \frac{g_{\mathrm{o}} r_{\mathrm{o}}}{c_{\mathrm{p}} T_{\infty}}\left(1-\frac{r_{\mathrm{o}}}{r_{\mathrm{m}}}\right)+1\right]^{\left(c_{\mathrm{p}} m / \mathrm{k}\right)}$

For $\left(g_{\mathrm{o}} r_{\mathrm{o}} / c_{\mathrm{p}}\right)=3400 \mathrm{~K}, T_{\infty}=1000 \mathrm{~K}, r_{\mathrm{o}} / r_{\mathrm{m}}=0.5$, and $c_{\mathrm{p}} m / \mathrm{k}=9 / 2$, I calculate $p_{\mathrm{o}} / p\left(r_{\mathrm{m}}\right)=87.3$. Under these conditions, $p\left(r_{\mathrm{m}}\right)=0.18 \mathrm{bar}$, so $p_{\mathrm{o}}=$ $15.7 \mathrm{bar}$. The surface pressure would have been higher for $r_{\mathrm{m}} / r_{\mathrm{o}}<2$. The upper limit, for the isothermal atmosphere at $1000 \mathrm{~K}$, is 481 bar.

The escape of this primary atmosphere, after dissipation of the solar nebula, would have been subject to the constraints described above concerning the rate of supply of energy to the thermosphere. Some remnants, particularly the heavier gases, may have remained behind to mix with volatiles released by degassing of the solid phase. 


\section{CONCLUSIONS}

In this paper I have explored some of the constraints imposed by atmospheric physics on the properties of the Earth's earliest atmosphere. These constraints are intended as a stimulus to further more careful consideration and not in any sense as a definitive statement of what this atmosphere was like. Some of my suggestions are probably wrong.

It appears that escape of massive amounts of gas into the interplanetary vacuum would have been possible, provided heating of the thermosphere by radiations from the young Sun was very much larger than it is today and provided, also, that the mean molecular mass of the atmosphere was small. This escape could have been unselective with respect to heavy minor constituents provided the escape rate was large enough. A decreasing escape rate caused, for example, by decreasing solar ultraviolet luminosity, would have permitted progressively lighter gases to be left behind by the escape process. It seems unlikely that the upper reaches of an atmosphere escaping into empty space would have been turbulent.

Of possibly greater relevance is the atmosphere that would have formed if the Earth had accreted and released most of its volatiles while still immersed in a gaseous nebula. If such an atmosphere were isothermal it would necessarily have been hot, to prevent an unduly large accumulation of mass and gravitational collapse of the nebula onto the protoplanet. An approximately isothermal and static atmosphere, however, would have permitted diffusive concentration of heavy gases from the nebula into the atmosphere. The concentration factors are so large and diffusion times so short that this model can probably be discarded.

A fully turbulent atmosphere with an adiabatic temperature profile at all levels yields unreasonably high surface temperatures. More plausible is a freely-convecting adiabatic region near the surface, overlain by a region of lower temperature gradient in which accretional energy is carried away from the planet by radiation. Rotation of the planet would impose on this upper region of the atmosphere a global circulation in which nebular gas descends over the poles and atmospheric gas flows outwards over the equator. The convective region of the atmosphere would probably rotate rigidly with the planet, while angular velocity would decrease rapidly in the overlying region, which was probably in laminar flow.

\section{ACKNOWLEDGEMENTS}

I have benefitted from the comments and suggestions of A.J. Watson and D.M. Hunten. P.B. Hays offered useful suggestions concerning fluid dynamics. This research was supported in part by the National Aeronautics and Space Administration under Grant NAGW-176. Much of the writing was performed while I was a UNESCO consultant in the Department of Physics at Andhra University, Waltair, India. I am grateful to the officials of Andhra University and to my colleagues there for their kind hospitality. 


\section{REFERENCES}

Anders, E. and Owen, T., 1977. Mars and Earth: origin and abundance of volatiles. Science, 198: 453-465.

Arculus, R.J. and Delano, J.W., 1980. Implications for the primitive atmosphere of the oxidation state of Earth's upper mantle. Nature (London), 288: 72-74.

Aston, F.W., 1924. The rarity of the inert gases on the Earth. Nature (London), 114: 786.

Banks, P.M. and Holzer, T.E., 1968. The polar wind. J. Geophys. Res., 73: 6846-6854.

Banks, P.M. and Holzer, T.E., 1969a. Features of plasma transport in the upper atmosphere. J. Geophys. Res., 74: 6304-6316.

Banks, P.M. and Holzer, T.E., 1969b. High-latitude plasma transport: the polar wind. J. Geophys. Res., 74: 6317-6332.

Brancazio, P.J. and Cameron, A.G.W. (Editors), 1963. The Origin and Evolution of Atmospheres and Oceans. John Wiley, New York, NY.

Brinkmann, R.T., 1970. Departures from Jeans' escape rate for $\mathrm{H}$ and $\mathrm{He}$ in the Earth's atmosphere. Planet. Space Sci., 18:449-478.

Brinkmann, R.T., 1971. More comments on the validity of Jeans' escape rate. Planet. Space Sci., 19: 791-794.

Brown, H., 1952. Rare gases and the formation of the Earth's atmosphere. In: G.P. Kuiper (Editor), The Atmospheres of the Earth and Planets. University of Chicago Press, Chicago, IL, 2nd edn., pp. 258-266.

Cameron, A.G.W. and Pine, M.R., 1973. Numerical models of the primitive solar nebula. Icarus, 18: 377-406.

Chamberlain, J.W., 1963. Planetary coronae and atmospheric evaporation. Planet. Space Sci., 11: $901-960$.

Chamberlain, J.W., 1969. Escape rate of hydrogen from a carbon dioxide atmosphere. Astrophys. J., 155: 711-714.

Chamberlain, J.W. and Campbell, F.J., 1967. Rate of evaporation of a non-Maxwellian atmosphere. Astrophys. J., 149: 687-705.

Chamberlain, J.W. and Smith, G.R., 1971. Comments on the rate of evaporation of a non-Maxwellian atmosphere. Planet. Space Sci., 19: 675-684.

Clark, S.P., Turekian, K.K. and Grossman, L., 1972. Model for the early history of the Earth. In: E.C. Robertson (Editor), The Nature of the Solid Earth. McGraw-Hill, New York, NY, pp. 3-18.

Fanale, F.P., 1971. A case for catastrophic early degassing of the Earth. Chem. Geol., 8: 79-105.

Gehrels, T. (Editor), 1978. Protostars and Planets. University of Arizona Press, Tucson, AZ.

Goldreich, P. and Ward, W.R., 1973. The formation of planetesimals. Astrophys. J., 183 1051-1061.

Goody, R.M., 1964. Atmospheric Radiation. Oxford University Press, London.

Goody, R.M. and Walker, J.C.G., 1972. Atmospheres. Prentice-Hall, Englewood Cliffs, NJ.

Grossman, L., 1972. Equilibrium condensation in the primitive solar nebula. Geochim. Cosmochim. Acta, 36: 597-619.

Grossman, L. and Larimer, J.W., 1974. Early chemical history of the solar system. Rev. Geophys. Space Phys., 12: 71-101.

Hanks, T.C. and Anderson, D.L., 1969. The early thermal history of the Earth. Phys. Earth Planet. Inter., 2: 19-29.

Hayashi, C., Nakazawa, K. and Mizuno, H., 1979. Earth's melting due to the blanketing effect of the primordial dense atmosphere. Earth Planet. Sci. Lett., 43: 22-28. 
Hays, P.B. and Liu, V.C., 1965. On the loss of gases from a planetary atmosphere. Planet. Space Sci., 13: 1185-1212.

Hess, L., 1959. Introduction to Theoretical Meteorology. Holt, Rinehart and Winston, New York, NY.

Hills, J.G., 1973. On the process of accretion in the formation of the planets and comets. Icarus, 18: 505-522.

Holzer, T.E., Fedder, J.A. and Banks, P.M., 1971. A comparison of kinetic and hydrodynamic models of an expanding ion-exosphere. J. Geophys. Res., 76: 2453-2468.

Hunten, D.M., 1973. The escape of light gases from planetary atmospheres. J. Atmos. Sci., 30: 1481-1494.

Hunten, D.M., 1979. Capture of Phobos and Deimos by protoatmospheric drag. Icarus, 37: $113-123$.

Hunten, D.M. and Donahue, T.M., 1976. Hydrogen loss from the terrestrial planets. Annu. Rev. Earth Planet. Sci., 4: 265-292.

Ingersoll, A.P., 1969. The runaway greenhouse: a history of water on Venus. J. Atmos. Sci., 26: 1191-1198.

Jastrow, R. and Cameron, A.G.W. (Editors), 1963. Origin of the Solar System. Academic Press, New York, NY.

Lamb, H., 1932. Hydrodynamics. Dover Publications, New York, NY.

Landau, L.D. and Lifshitz, E.M., 1959. Fluid Mechanics. Pergamon, Oxford.

Larimer, J.W., 1973. Chemistry of the solar nebula. Space Sci. Rev., 15: 103-119.

Lewis, J.S., 1972a. Metal silicate fractionation in the solar system. Earth Planet. Sci. Lett., 15: 286-290.

Lewis, J.S., 1972b. Low-temperature condensation from the solar nebula. Icarus, 16: 241-252.

Lewis, J.S., 1974a. The temperature gradient in the solar nebula. Science, 186: 440-443.

Lewis, J.S., 1974b. The chemistry of the solar system. Sci. Am., 230: 51-65.

MacDonald, G.J.F., 1959. Calculations on the thermal history of the Earth. J. Geophys. Res., 64: 1967-2000.

Meadows, A.J., 1973. The origin and evolution of the atmospheres of the terrestrial planets. Planet. Space Sci., 21 : 1467-1474.

Mizuno, H., Nakazawa, K. and Hayashi, C., 1978. Instability of a gaseous envelope surrounding a planetary core and formation of giant planets. Prog. Theor. Phys., 60: 699-710.

Mizuno, H., Nakazawa, K. and Hayashi, C., 1980. Dissolution of the primordial rare gases into the molten Earth's mantle. Earth Planet. Sci. Lett., 50: 202-210.

Moulton, F.R., 1905. On the evolution of the solar system. Astrophys. J., 22: 165-181.

Parker, E.N., 1958. Dynamics of the interplanetary gas and magnetic fields. Astrophys. J., 128: 664 .

Parker, E.N., 1964. Coronal expansion and solar corpuscular radiation. In: H. Odishaw (Editor), Research in Geophysics, Vol. 1. M.I.T. Press, Cambridge, MA, pp. 99-114.

Parker, E.N., 1971. Recent developments in theory of solar wind. Rev. Geophys. Space Phys., 9: $825-835$.

Pollack, J.B. and Black, D.C., 1979. Implications of the gas compositional measurements of pioneer Venus for the origin of planetary atmospheres. Science, 205: 56-59.

Pollack, J.B. and Yung, Y.L., 1980. Origin and evolution of planetary atmospheres. Annu. Rev. Earth Planet. Sci., 8: 425-487.

Ringwood, A.E., 1979. Origin of the Earth and Moon. Springer-Verlag, New York, NY.

Russel, H.N. and Menzel, D.H., 1933. The terrestrial abundance of the permanent gases. Proc. U.S. Natl. Acad. Sci., 19: 997-1001.

Sagan, C., 1967. Origins of atmospheres of Earth and planets. In: S.K. Runcorn (Editor), International Dictionary of Geophysics, Vol. 1. Pergamon, New York, NY. pp. 97-104.

Schlichting, H., 1955. Boundary Layer Theory. Pergamon, New York, NY. 
Sekiya, M., Nakazawa, K. and Hayashi, C., 1980. Dissipation of the rare gases contained in the primordial Earth's atmosphere. Earth Planet. Sci. Lett., 50: 197-201.

Stevenson, D.J., 1982. The nature of the Earth prior to the rock record (the Hadean Earth). In: J.W. Schopf (Editor), The Earth's Earliest Biosphere: its Origin and Evolution. Princeton University Press, Princeton, NJ (in press).

Strobel, D.F. and Weber, E.J., 1972. Mathematical model. of the polar wind. J. Geophys. Res., 77: 6864-6869.

Strom, S.E., 1977. Early stages of stellar evolution. In: T. de Jong and A. Maeder (Editors), Star Formation. Proc. IAU Symp. No. 75, pp. 179-212.

Suess, H.E., 1949. Die Häufigkeit der Edelgase auf der Erde und im Kosmos. J. Geol., 57: 600 .

Torr, M.R., Richards, P.G. and Torr, D.G., 1980. A new determination of the ultraviolet heating efficiency of the thermosphere. J. Geophys. Res., 85: 6819-6826.

Turekian, K.K. and Clark, S.P., 1969. Inhomogeneous accumulation of the Earth from the primitive solar nebula. Earth Planet. Sci. Lett., 6: 346-348.

Turekian, K.K. and Clark, S.P., 1975. The non-homogeneous accumulation model for terrestrial planet formation and the consequences for the atmosphere of Venus. J. Atmos. Sci., 32: 1257-1261.

Urey, H.C.; 1952. The Planets. Yale University Press, New Haven, CT.

Walker, J.C.G., 1977. Evolution of the Atmosphere. Macmillan, New York, NY.

Watson, A.J., Donahue, T.M. and Walker, J.C.G., 1981. Dynamics of a rapidly escaping atmosphere: applications to the evolution of Earth and Venus. Icarus, 48: 150-166.

Weast, R.C. (Editor), 1978. CRC Handbook of Chemistry and Physics. CRC Press, West Palm Beach, FL.

Weidenschilling, S.J., 1974. A model for the accretion of the terrestrial planets I. Icarus, 22: $426-435$.

Weidenschilling, S.J., 1976. Accretion of the terrestrial planets. II. Icarus, 27: 161-170.

Yeh, T., 1970. A three-fluid model of solar winds. Planet. Space Sci., 18: 199-215.

Zahnle, K.J. and Walker, J.C.G., 1982. The evolution of solar ultraviolet luminosity. Rev. Geophys. Space Phys. (submitted for publication). 